\title{
The Oresme sequence: The generalization of its matrix form and its hybridization process
}

\author{
Milena Carolina dos Santos Mangueira ${ }^{1}$, \\ Renata Passos Machado Vieira ${ }^{2}$, Francisco Regis Vieira Alves ${ }^{3}$ \\ and Paula Maria Machado Cruz Catarino ${ }^{4}$ \\ ${ }^{1}$ Department of Mathematics, Federal Institute of Education, Science and Technology \\ of State of Ceara - IFCE, Treze of Maio, Brazil \\ e-mail: milenacarolina24@gmail.com \\ ${ }^{2}$ Department of Mathematics, Federal Institute of Education, Science and Technology \\ of State of Ceara - IFCE, Treze of Maio, Brazil \\ e-mail: re.passosmegmail.com \\ ${ }^{3}$ Department of Mathematics, Federal Institute of Education, Science and Technology \\ of State of Ceara - IFCE, Treze of Maio, Brazil \\ e-mail: fregisdifce.edu.br \\ ${ }^{4}$ University of Trás-os-Montes and Alto Douro - UTAD \\ Vila Real, Portugal \\ e-mail: pcatarino23@gmail.com
}

\begin{abstract}
This article deals with the generalization of the matrix form of the Oresme sequence, extending it to the field of integers. In addition, the two valid generating matrices were discussed, through the permutation of the rows and columns, respectively, totaling two valid matrices of Oresme for the left-hand side, and two more generating matrices of Oresme for the right-hand side. In addition, a new Oresme sequence was introduced, given through the hybridization process of these numbers, obtaining mathematical properties and theorems, inherent to this process.
\end{abstract}

Keywords: Matrix form of Oresme, Oresme's hybrid numbers, Oresme sequence.

2010 Mathematics Subject Classification: 11B37, 11B39, $11 \mathrm{~B} 83$. 


\section{Introduction}

The Oresme sequence was introduced by Nicole Oresme (1320-1382) in the 14-th century. The cleric found the sum of the rational numbers formed by the terms $\frac{1}{2}, \frac{2}{4}, \frac{3}{8}, \frac{4}{16}, \frac{5}{32}, \cdots, \frac{n}{2^{n}}$, these numbers form a second order sequence, studied in $[6,7,11]$, which has its recurrence formula derived from the Fibonacci sequence, be it: $O_{n+2}=O_{n+1}-\frac{1}{4} O_{n}, n \geq 0$, in which, $O_{0}=0$ and $O_{1}=\frac{1}{2}$ are the initial conditions. In [11], Horadam comments that he can determine Oresme's numbers as: $O_{n}=-n \cdot 2^{n}$ with $n$ being a positive integer.

In order to explore the mathematical process, there is then the generalization of the matrix form of this sequence, evolving in mathematical investigation. In addition, the generating matrices of the Oresme sequence will be presented, making it possible to obtain the terms of a linear and recurring sequence, without the need to calculate their previous terms.

In addition, the complexification process begins, carrying out an application of the hybrid numbers in this Oresme sequence.

In order to explore this sequence, its hybridization process will be carried out, that is, relate the set of hybrid numbers to this sequence [3, 5, 13-15, 17, 19, 20].

The set of hybrid numbers, denoted by $\mathbf{K}$, was presented by Özdemir [16] and, therefore, three numerical systems being combined, complex, hyperbolic and dual numbers are studied. In addition, Carvalho [4] presents a two-dimensional geometric representation of such numbers using a software.

This set is defined by:

Definition 1.1. A hybrid number is defined as:

$$
\begin{aligned}
\mathbf{K}=\{z=a+b i+c \varepsilon+d h \mid & a, b, c, d \in R, \\
& \left.i^{2}=-1, \varepsilon^{2}=0, h^{2}=1, i h=-h i=\varepsilon+i\right\}
\end{aligned}
$$

It enables us to perform operations on these numbers, whether they are: adding or subtracting hybrid numbers, multiplying a scalar with a hybrid number, or multiplying two hybrid numbers. The character of the real number can also be presented $C(z)=z \bar{z}=\bar{z} z=a^{2}+(b-c)^{2}-c^{2}-d^{2}=$ $a^{2}+b^{2}-2 b c-d^{2}$, where the root of that real number will be the norm of the hybrid number $z$, so we have to: $\|z\|=\sqrt{|C(z)|}$.

Furthermore, the Oresme generating matrices will be explored, presenting properties inherent to them, as well as their extension to the field of integers. With that, in the following sections we will introduce a new definition called: Oresme's hybrid numbers. We will study its recurrence, characteristic equation, norm of the hybrid number of Oresme, matrix form, generating function and its extension for negative indices.

\section{Generating matrices of Oresme}

In order to complement the study regarding the generalization process of the Oresme sequence, we have the generalization of the matrix form of these numbers, as a way to carry out the mathematical evolution process of this sequence. 
Linear and recurring sequences have a way of obtaining the terms of their sequence or need to know the previous terms, denominated in a matrix form or even a generating matrix [10]. Based on the generating matrix of the $k$-Oresme [7] sequence, we can replace the value of $k$ with 2 , thus obtaining the generating matrix of the Oresme sequence.

Theorem 2.1. The Oresme generating matrix is given by [1]. For $O=\left[\begin{array}{cc}1 & -\frac{1}{4} \\ 1 & 0\end{array}\right]$ we have that: $O^{n}=\left[\begin{array}{cc}2 O_{n+1} & -\frac{1}{2} O_{n} \\ 2 O_{n} & -\frac{1}{2} O_{n-1}\end{array}\right]^{n}, n \geqslant 1$.

Proof. Using the principle of finite induction, we have for $n=1$ :

$$
O=\left[\begin{array}{cc}
1 & -\frac{1}{4} \\
1 & 0
\end{array}\right]=\left[\begin{array}{cc}
2\left(\frac{2}{4}\right) & -\frac{1}{2}\left(\frac{1}{2}\right) \\
2\left(\frac{1}{2}\right) & -\frac{1}{2}(0)
\end{array}\right]=\left[\begin{array}{cc}
2 O_{2} & -\frac{1}{2} O_{1} \\
2 O_{1} & -\frac{1}{2} O_{0}
\end{array}\right]
$$

So the equality is valid.

Assuming it is valid for $n=k, k \in \mathbb{N}$, we have:

$$
O^{k}=\left[\begin{array}{cc}
1 & -\frac{1}{4} \\
1 & 0
\end{array}\right]^{k}=\left[\begin{array}{cc}
2 O_{k+1} & -\frac{1}{2} O_{k} \\
2 O_{k} & -\frac{1}{2} O_{k-1}
\end{array}\right]
$$

Thus, it will also be valid for $n=k+1$ :

$$
\begin{aligned}
O^{k+1} & =\left[\begin{array}{cc}
1 & -\frac{1}{4} \\
1 & 0
\end{array}\right]^{k+1}=\left[\begin{array}{cc}
1 & -\frac{1}{4} \\
1 & 0
\end{array}\right]^{k}\left[\begin{array}{cc}
1 & -\frac{1}{4} \\
1 & 0
\end{array}\right] \\
& =\left[\begin{array}{cc}
2 O_{k+1} & -\frac{1}{2} O_{k} \\
2 O_{k} & -\frac{1}{2} O_{k-1}
\end{array}\right]\left[\begin{array}{cc}
1 & -\frac{1}{4} \\
1 & 0
\end{array}\right] \\
& =\left[\begin{array}{ll}
2 O_{k+1}-\frac{1}{2} O_{k} & -\frac{1}{2} O_{k+1} \\
2 O_{k}-\frac{1}{2} O_{k-1} & -\frac{1}{2} O_{k}
\end{array}\right] \\
& =\left[\begin{array}{ll}
2 O_{k+2} & -\frac{1}{2} O_{k+1} \\
2 O_{k+1} & -\frac{1}{2} O_{k}
\end{array}\right]
\end{aligned}
$$

Therefore, we can perform the permutation of rows and columns of the $O$ generating matrix, obtaining another matrix from the Oresme sequence, totaling two valid matrices.

Theorem 2.2. For $O=\left[\begin{array}{cc}0 & 1 \\ -\frac{1}{4} & 1\end{array}\right]$, we have that: $O^{n}=\left[\begin{array}{cc}-\frac{1}{2} O_{n-1} & 2 O_{n} \\ -\frac{1}{2} O_{n} & 2 O_{n+1}\end{array}\right]^{n}, n \geqslant 1$.

Proof. Analogously to Theorem 2.1, we can validate this theorem.

In the work of Horadam [11], it is reported that Oresme's numbers can be extended to the left-hand side, field of whole numbers, observing the behavior of the sequence it is noticed that an integer number of Oresme is defined by $O_{n}=-n \cdot 2^{n}, n>0$. Therefore, we will introduce the inverse matrices, based on the work of Alves [1].

In order to explore the process of extending these numbers to negative integer terms, we can perform some algebraic manipulations in their original recurrence. 
Definition 2.3. The Oresme sequence recurrence formula, for the terms on the left-hand side, for $n \in \mathbb{N}$, is given by:

$$
O_{-n}=4 O_{-n+1}-4 O_{-n+2}, n<0 .
$$

So, we have then the terms on the left-hand side as shown in the Table 1.

\begin{tabular}{|c|c|c|c|c|c|c|c|c|c|}
\hline$O_{-1}$ & $O_{-2}$ & $O_{-3}$ & $O_{-4}$ & $O_{-5}$ & $O_{-6}$ & $O_{-7}$ & $O_{-8}$ & $O_{-9}$ & $O_{-10}$ \\
\hline-2 & -8 & -24 & -64 & -160 & -384 & -896 & -2048 & -4608 & -10240 \\
\hline
\end{tabular}

Table 1: Negative integer terms of the Oresme sequence, of the type $O_{-n}$.

With this, we have the theorems inherent to Oresme's inverse matrices, emphasizing the generalization process of these matrices. For this, it was necessary to calculate the inverse of the base matrix, calling it $\sigma$.

Theorem 2.4. The generating matrix form of Oresme for the field of negative integers, is given by:

$$
\text { For } \sigma=\left[\begin{array}{cc}
0 & 1 \\
-4 & 4
\end{array}\right] \text {, we have to: } \sigma^{n}=\left[\begin{array}{cc}
2 O_{-n+1} & -\frac{1}{2} O_{-n} \\
2 O_{-n} & -\frac{1}{2} O_{-n-1}
\end{array}\right]^{n} \text {, for } n>0
$$

Proof. Using the same principle performed previously, finite induction, for $n=1$, we have:

$$
\sigma=\left[\begin{array}{cc}
0 & 1 \\
-4 & 4
\end{array}\right]=\left[\begin{array}{cc}
2(0) & -\frac{1}{2}(-2) \\
2(-2) & -\frac{1}{2}(-8)
\end{array}\right]=\left[\begin{array}{cc}
2 O_{0} & -\frac{1}{2} O_{-1} \\
2 O_{-1} & -\frac{1}{2} O_{-2}
\end{array}\right]
$$

So the equality is valid.

Assuming it is valid for $n=k, k \in \mathbb{N}$, we have:

$$
\sigma^{k}=\left[\begin{array}{cc}
0 & 1 \\
-4 & 4
\end{array}\right]^{k}=\left[\begin{array}{cc}
2 O_{-k+1} & -\frac{1}{2} O_{-k} \\
2 O_{-k} & -\frac{1}{2} O_{-k-1}
\end{array}\right]
$$

Now, we will verify that it is valid for $n=k+1$, we have:

$$
\begin{aligned}
\sigma^{k+1} & =\left[\begin{array}{cc}
0 & 1 \\
-4 & 4
\end{array}\right]^{k+1}=\left[\begin{array}{cc}
0 & 1 \\
-4 & 4
\end{array}\right]^{k}\left[\begin{array}{cc}
0 & 1 \\
-4 & 4
\end{array}\right] \\
& =\left[\begin{array}{cc}
2 O_{-k+1} & -\frac{1}{2} O_{-k} \\
2 O_{-k} & -\frac{1}{2} O_{-k-1}
\end{array}\right]\left[\begin{array}{cc}
0 & 1 \\
-4 & 4
\end{array}\right] \\
& =\left[\begin{array}{cc}
2 O_{-k} & 2 O_{-k+1}-2 O_{-k} \\
2 O_{-k-1} & 2 O_{-k}-2 O_{-k-1}
\end{array}\right] \\
& =\left[\begin{array}{cc}
2 O_{-k} & -\frac{1}{2} O_{-k-1} \\
2 O_{-k-1} & -\frac{1}{2} O_{-k-2}
\end{array}\right] .
\end{aligned}
$$

In a similar way, we can obtain another Oresme generating matrix, exchanging the rows and columns, respectively.

Theorem 2.5. For $\sigma=\left[\begin{array}{cc}4 & -4 \\ 1 & 0\end{array}\right]$, we have: $\sigma^{n}=\left[\begin{array}{cc}-\frac{1}{2} O_{-n-1} & 2 O_{-n} \\ -\frac{1}{2} O_{-n} & 2 O_{-n+1}\end{array}\right]$, for $n>0$.

Proof. Similar to the proof of Theorem 2.4, this theorem can be validated. 


\subsection{Properties inherent to matrices}

It is possible to establish some properties regarding the matrices previously encountered.

Property 2.6. For any integer $m, r, 0<m<r$, we have:

$$
O_{r+2}=2 O_{m} O_{r-m+2}-\frac{1}{2} O_{m} O_{r}
$$

and

$$
O_{r+2}=-\frac{1}{2} O_{m-2} O_{r-m+2}+\frac{1}{8} O_{m-2} O_{r} .
$$

Proof. According to Theorem 2.1 and some properties for square matrix exponents, we have:

$$
\begin{aligned}
O^{r+2}= & O^{m-1} O^{r-m+2}-\frac{1}{4} \cdot O^{m-1} O^{r} \\
{\left[\begin{array}{ll}
2 O_{r+3} & -\frac{1}{2} O_{r+2} \\
2 O_{r+2} & -\frac{1}{2} O_{r+1}
\end{array}\right]=} & {\left[\begin{array}{cc}
2 O_{m} & -\frac{1}{2} O_{m-1} \\
2 O_{m-1} & -\frac{1}{2} O_{m-2}
\end{array}\right]\left[\begin{array}{cc}
2 O_{r-m+3} & -\frac{1}{2} O_{r-m+2} \\
2 O_{r-m+2} & -\frac{1}{2} O_{r-m+1}
\end{array}\right] } \\
& -\frac{1}{4}\left[\begin{array}{cc}
2 O_{m} & -\frac{1}{2} O_{m-1} \\
2 O_{m-1} & -\frac{1}{2} O_{m-2}
\end{array}\right]\left[\begin{array}{cc}
2 O_{r+1} & -\frac{1}{2} O_{r} \\
2 O_{r} & -\frac{1}{2} O_{r-1}
\end{array}\right] .
\end{aligned}
$$

Considering the left and right elements, we have:

$$
O_{r+2}=2 O_{m} O_{r-m+2}-\frac{1}{2} O_{m} O_{r}
$$

and

$$
O_{r+2}=-\frac{1}{2} O_{m-2} O_{r-m+2}+\frac{1}{8} O_{m-2} O_{r} .
$$

Note 2.7. On Property 2.1, if $m=1$, we have:

$$
\begin{aligned}
O_{r+2} & =2 O_{m} O_{r-m+2}-\frac{1}{2} O_{m} O_{r} \\
O_{r+2} & =2 O_{1} O_{r-1+2}-\frac{1}{2} O_{1} O_{r} \\
O_{r+2} & =2 \cdot \frac{1}{2} O_{r+1}-\frac{1}{2} \cdot \frac{1}{2} O_{r} \\
O_{r+2} & =O_{r+1}-\frac{1}{4} O_{r} .
\end{aligned}
$$

Property 2.8. For any integer $m, r, 0<m<r$, we have to:

$$
O_{r+2}=-\frac{1}{2} O_{m-2} O_{r+1}-\frac{1}{2} O_{r} O_{m}
$$

and

$$
O_{r+2}=2 O_{m} O_{r+1}+\frac{1}{8} O_{r} O_{m-2}
$$

Proof. According to Theorem 2.2 and some properties for square matrix exponents, we have:

$$
\begin{aligned}
O^{r+2}= & O^{m-1} O^{r+1}-\frac{1}{4} O^{r} O^{m-1} \\
{\left[\begin{array}{cc}
-\frac{1}{2} O_{r+1} & 2 O_{r+2} \\
-\frac{1}{2} O_{r+2} & 2 O_{r+3}
\end{array}\right]=} & {\left[\begin{array}{cc}
-\frac{1}{2} O_{m-2} & 2 O_{m-1} \\
-\frac{1}{2} O_{m-1} & 2 O_{m}
\end{array}\right]\left[\begin{array}{cc}
-\frac{1}{2} O_{r} & 2 O_{r+1} \\
-\frac{1}{2} O_{r+1} & 2 O_{r+2}
\end{array}\right] } \\
& -\frac{1}{4}\left[\begin{array}{cc}
-\frac{1}{2} O_{r-1} & 2 O_{r} \\
-\frac{1}{2} O_{r} & 2 O_{r+1}
\end{array}\right]\left[\begin{array}{cc}
-\frac{1}{2} O_{m-2} & 2 O_{m-1} \\
-\frac{1}{2} O_{m-1} & 2 O_{m}
\end{array}\right] .
\end{aligned}
$$

Considering the left and right elements, we have:

$$
O_{r+2}=-\frac{1}{2} O_{m-2} O_{r+1}-\frac{1}{2} O_{r} O_{m}
$$

and

$$
O_{r+2}=2 O_{m} O_{r+1}+\frac{1}{8} O_{r} O_{m-2} .
$$


Note 2.9. On Property 2.3, if $m=1$, we have to:

$$
\begin{aligned}
O_{r+2} & =-\frac{1}{2} O_{m-2} O_{r+1}-\frac{1}{2} O_{r} O_{m} \\
O_{r+2} & =-\frac{1}{2} O_{-1} O_{r+1}-\frac{1}{2} O_{r} O_{1} \\
O_{r+2} & =-\frac{1}{2}(-2) O_{r+1}-\frac{1}{2} \cdot \frac{1}{2} O_{r} \\
O_{r+2} & =O_{r+1}-\frac{1}{4} O_{r} .
\end{aligned}
$$

Now, by exploring the properties for terms with negative integer indices, the following results can be obtained.

Property 2.10. For any integer $m, r, 0<m<r$, we have:

$$
O_{-r}=-2 O_{-m} O_{-r+1}+2 O_{-m} O_{r+2}
$$

and

$$
O_{-r}=-8 O_{-m} O_{-r+4}+8 O_{-m} O_{-r+3} .
$$

Proof. According to Theorem 2.4 and some properties for square matrix exponents, we have:

$$
\begin{aligned}
\sigma^{r}= & 4 \sigma^{m} \sigma^{r-2}-4 \sigma^{m} \sigma^{r-3} \\
{\left[\begin{array}{cc}
2 O_{-r+1} & -\frac{1}{2} O_{-r} \\
2 O_{-r} & -\frac{1}{2} O_{-r-1}
\end{array}\right]=} & 4\left[\begin{array}{cc}
2 O_{-m+1} & -\frac{1}{2} O_{-m} \\
2 O_{-m} & -\frac{1}{2} O_{-m-1}
\end{array}\right]\left[\begin{array}{ll}
2 O_{-r+3} & -\frac{1}{2} O_{-r+2} \\
2 O_{-r+2} & -\frac{1}{2} O_{r+1}
\end{array}\right] \\
& -4\left[\begin{array}{cc}
2 O_{-m+1} & -\frac{1}{2} O_{-m} \\
2 O_{-m} & -\frac{1}{2} O_{-m-1}
\end{array}\right]\left[\begin{array}{ll}
2 O_{-r+4} & -\frac{1}{2} O_{-r+3} \\
2 O_{-r+3} & -\frac{1}{2} O_{r+2}
\end{array}\right] .
\end{aligned}
$$

Considering the left and right elements, we have:

$$
O_{-r}=-2 O_{-m} O_{-r+1}+2 O_{-m} O_{r+2}
$$

and

$$
O_{-r}=-8 O_{-m} O_{-r+4}+8 O_{-m} O_{-r+3} .
$$

Note 2.11. In Property 2.5, if $m=1$, we have:

$$
\begin{aligned}
& O_{-r}=-2 O_{-m} O_{-r+1}+2 O_{-m} O_{r+2} \\
& O_{-r}=-2 O_{-1} O_{-r+1}+2 O_{-1} O_{r+2} \\
& O_{-r}=-2(-2) O_{-r+1}+2(-2) O_{r+2} \\
& O_{-r}=4 O_{-r+1}-4 O_{r+2} .
\end{aligned}
$$

Property 2.12. For any integer $m, r, 0<m<r$, we have:

$$
O_{-r}=-2 O_{-m} O_{-r+1}+2 O_{-m} O_{r+2}
$$

and

$$
O_{-r}=-O_{-r-1}+O_{-r}
$$

Proof. According to Theorem 2.5 and some properties for square matrix exponents, we have:

$$
\begin{aligned}
\sigma^{r}= & -\sigma^{m} \sigma^{r}+\sigma^{m} \sigma^{r-1} \\
{\left[\begin{array}{cc}
-\frac{1}{2} O_{-r+1} & 2 O_{-r} \\
-\frac{1}{2} O_{-r} & 2 O_{-r+1}
\end{array}\right]=} & -\left[\begin{array}{cc}
-\frac{1}{2} O_{-m-1} & 2 O_{-m} \\
-\frac{1}{2} O_{-m} & 2 O_{-m+1}
\end{array}\right]\left[\begin{array}{cc}
-\frac{1}{2} O_{-r-1} & 2 O_{-r} \\
-\frac{1}{2} O_{-r} & 2 O_{-r+1}
\end{array}\right] \\
+ & {\left[\begin{array}{cc}
-\frac{1}{2} O_{-m-1} & 2 O_{-m} \\
-\frac{1}{2} O_{-m} & 2 O_{-m+1}
\end{array}\right]\left[\begin{array}{cc}
-\frac{1}{2} O_{-r} & 2 O_{-r+1} \\
-\frac{1}{2} O_{-r+1} & 2 O_{-r+2}
\end{array}\right] }
\end{aligned}
$$


Considering the left and right elements, we have:

$$
O_{-r}=-2 O_{-m} O_{-r+1}+2 O_{-m} O_{r+2}
$$

and

$$
O_{-r}=-O_{-r-1}+O_{-r}
$$

Note 2.13. In Property 2.7, if $m=1$, we have:

$$
\begin{aligned}
& O_{-r}=-2 O_{-m} O_{-r+1}+2 O_{-m} O_{r+2} \\
& O_{-r}=-2 O_{-1} O_{-r+1}+2 O_{-1} O_{r+2} \\
& O_{-r}=-2(-2) O_{-r+1}+2(-2) O_{r+2} \\
& O_{-r}=-4 O_{-r+1}-4 O_{r+2} .
\end{aligned}
$$

\section{Oresme's hybrid numbers}

In this section, we will define a new type of hybrid number, the Oresme's hybrid numbers, and some results obtained from that definition.

Definition 3.1. The hybrid number of Oresme, called $H O_{n}, n \in \mathbb{R}$, is defined as:

$$
H O_{n}=O_{n}+O_{n+1} i+O_{n+2} \varepsilon+O_{n+3} h
$$

with the following initial conditions: $H O_{0}=\frac{1}{2} i+\frac{2}{4} \varepsilon+\frac{3}{8} h$ and $H O_{1}=\frac{1}{2}+\frac{2}{4} i+\frac{3}{8} \varepsilon+\frac{4}{16} h$.

On the other hand, it can be said that Oresme's hybrid numbers can also be described as:

$$
H O_{n}=\frac{n}{2^{n}}+\frac{n+1}{2^{n+1}} i+\frac{n+2}{2^{n+2}} \varepsilon+\frac{n+3}{2^{n+3}} h, n \geq 0 .
$$

Proposition 3.2. Oresme's hybrid number, $\mathrm{HO}_{n}$, satisfies

$$
H O_{n+2}=H O_{n+1}-\frac{1}{4} H O_{n}, \quad n \geq 0 .
$$

Proof. $H O_{n+1}-\frac{1}{4} H O_{n}$

$$
\begin{aligned}
& =\left(O_{n+1}+O_{n+2} i+O_{n+3} \varepsilon+O_{n+4} h\right)-\frac{1}{4}\left(O_{n}+O_{n+1} i+O_{n+2} \varepsilon+O_{n+3} h\right) \\
& =\left(O_{n+1}-\frac{1}{4} O_{n}\right)+\left(O_{n+2}-\frac{1}{4} O_{n+1}\right) i+\left(O_{n+3}-\frac{1}{4} O_{n+2}\right) \varepsilon+\left(O_{n+4}-\frac{1}{4} O_{n+3}\right) h \\
& =O_{n+2}+O_{n+3} i+O_{n+4} \varepsilon+O_{n+5} h \\
& =H O_{n+2} .
\end{aligned}
$$

According to the recurrence relationships, $\mathrm{HO}_{n+2}=H O_{n+1}-\frac{1}{4} H O_{n}$, you can present your characteristic equation, obtaining: $\frac{H O_{n+2}}{H O_{n+1}}=1-\frac{1}{4} \frac{H O_{n}}{H O_{n+1}}$. Using the reasoning carried out by 12 , the behavior of the convergence of the quotients is conjectured as being of the type $\frac{H O_{n}}{H O_{n-1}}>0$. So, we have to $\frac{H O_{n+2}}{H O_{n+1}}=1-\frac{1}{4} \frac{1}{\frac{H O_{n+1}}{H O_{n}}}$. Denoting $x_{n}=\frac{H O_{n+2}}{H O_{n+1}}$, we have: $x_{n-1}=\frac{H O_{n+1}}{H O_{n}}$. Determining the equation $x_{n}=1-\frac{1}{4} \cdot \frac{1}{x_{n-1}}$, where passing the limit and making the $n$ tend to infinity in this last expression, we have: 


$$
\begin{aligned}
\lim _{n \rightarrow \infty} x_{n} & =1-\frac{1}{4} \cdot \frac{1}{\lim _{n \rightarrow \infty} x_{n-1}} \\
x & =x-\frac{1}{4} \cdot \frac{1}{x} \\
4 x^{2}-4 x+1 & =0 .
\end{aligned}
$$

Thus determining the characteristic equation of an Oresme hybrid number, where it is a second degree equation having two real roots $x_{1}=x_{2}=\frac{1}{2}$. Based on what has been previously presented, we have that the character of the hybrid number of Oresme is given as $C\left(H O_{n}\right)=$ $O_{n}^{2}+\left(O_{n+1}-O_{n+2}\right)^{2}-O_{n+2}^{2}-O_{n+3}^{2}$. With this, the norm of a hybrid number of Oresme can be presented.

Proposition 3.3. The norm of an Oresme hybrid number is given by:

$$
\left\|H O_{n}\right\|^{2}=\left|O_{n}^{2}+\frac{15}{16} O_{n+1}^{2}-\frac{3}{2} O_{n+1} O_{n+2}-O_{n+2}^{2}\right| .
$$

Proof. $\left\|H O_{n}\right\|=\sqrt{\left|C\left(H O_{n}\right)\right|}$

$$
\begin{aligned}
\left\|H O_{n}\right\|^{2} & =\left|O_{n}^{2}+\left(O_{n+1}-O_{n+2}\right)^{2}-O_{n+2}^{2}-O_{n+3}^{2}\right| \\
& =\left|O_{n}^{2}+O_{n+1}^{2}-2 O_{n+1} O_{n+2}-O_{n+2}^{2}+\frac{1}{2} O_{n+1} O_{n+2}-\frac{1}{16} O_{n+1}^{2}\right| \\
& =\left|O_{n}^{2}+\frac{15}{16} O_{n+1}^{2}-\frac{3}{2} O_{n+1} O_{n+2}-O_{n+2}^{2}\right| .
\end{aligned}
$$

Definition 3.4. The hybrid vector of Oresme is a vector of a Minkowski space $E_{1}^{3}$, for $n \in \mathbb{N}$, defined by:

$$
\xi_{H O_{n}}=\left(\left(O_{n+1}-O_{n+2}\right), O_{n+2}, O_{n+3}\right) .
$$

Depending on its type, the hybrid number can be classified as elliptical (complike), hyperbolic (hyperlike) or parabolic (duallike) if $C_{\xi}\left(H O_{n}\right)<0, C_{\xi}\left(H O_{n}\right)>0$ or $C_{\xi}\left(H O_{n}\right)=0$, respectively. And yet, there is the matrix of Oresme's hybrid numbers that can be represented by a $2 \times 2$ matrix.

Proposition 3.5. A matrix of the hybrid number of Oresme, with $n \in \mathbf{N}$, is given by:

$$
\varphi_{H O_{n}}=\left[\begin{array}{cc}
O_{n}+O_{n+2} & \frac{3}{4} O_{n+1} \\
2 O_{n+2}-\frac{5}{4} O_{n+1} & O_{n}-O_{n+2}
\end{array}\right] .
$$

Proof. In [16] the matrix form of a hybrid number is defined as:

$$
\varphi_{a+b i+c \varepsilon+d h}=\left[\begin{array}{cc}
a+c & b-c+d \\
c-b+d & a-c
\end{array}\right] .
$$

Making the necessary substitutions, we have:

$$
\begin{aligned}
\varphi_{H O_{n}} & =\left[\begin{array}{cc}
O_{n}+O_{n+2} & O_{n+1}-O_{n+2}+O_{n+3} \\
O_{n+2}-O_{n+1}+O_{n+3} & O_{n}-O_{n+2}
\end{array}\right] \\
& =\left[\begin{array}{cc}
O_{n}+O_{n+2} & O_{n+1}-O_{n+2}+O_{n+2}-\frac{1}{4} O_{n+1} \\
O_{n+2}-O_{n+1}+O_{n+2}-\frac{1}{4} O_{n+1} & O_{n}-O_{n+2}
\end{array}\right] \\
& =\left[\begin{array}{cc}
O_{n}+O_{n+2} & \frac{3}{4} O_{n+1} \\
2 O_{n+2}-\frac{5}{4} O_{n+1} & O_{n}-O_{n+2}
\end{array}\right] .
\end{aligned}
$$


The following proposition that relates the determinant of a matrix to its norm can be presented.

Proposition 3.6. If $\varphi_{H_{n}}$ corresponds to the hybrid matrix of the Oresme's hybrid number, $H O_{n}$, then $\left\|H O_{n}\right\|^{2}=\operatorname{det}\left(\varphi_{H O_{n}}\right)$.

$$
\begin{aligned}
\text { Proof. } \operatorname{det}\left(\varphi_{H O_{n}}\right) & =\left|\begin{array}{cc}
O_{n}+O_{n+2} & \frac{3}{4} O_{n+1} \\
2 O_{n+2}-\frac{5}{4} O_{n+1} & O_{n}-O_{n+2}
\end{array}\right| \\
& =\left|\left(O_{n}+O_{n+2}\right)\left(O_{n}-O_{n+2}\right)-\frac{3}{4} O_{n+1}\left(2 O_{n+2}-\frac{5}{4} O_{n+1}\right)\right| \\
& =\left|O_{n}^{2}-O_{n+2}^{2}-\frac{3}{2} O_{n+1} O_{n+2}+\frac{15}{16} O_{n_{1}}^{2}\right| \\
& =\left\|H O_{n}\right\|^{2} .
\end{aligned}
$$

The following is a theorem inherent to Oresme's hybrid numbers.

Theorem 3.7. The generating function of the generalized hybrid number of Oresme, denoted by $G_{H O_{n}}(x)$, is:

$$
G_{H O_{n}}(x)=\frac{H O_{0}+\left(H O_{1}-H O_{0}\right) x}{\left(1-x+\frac{1}{4} x^{2}\right)}
$$

Proof. To define the generating function of the hybrid number of Oresme, denoted by $G\left(H O_{n}\right)(x)$, we will write a sequence in which each term corresponds to the coefficients:

$$
G_{H O_{n}}(x)=\sum_{n=0}^{\infty} H O_{n} x^{n} .
$$

Making algebraic manipulations due to the recurrence relationship, we can write this sequence as:

$$
\begin{aligned}
G_{H O_{n}}(x) & =H O_{0}+H O_{1} x+H O_{2} x^{2}+\cdots+H O_{n} x^{n}+\cdots \\
-x \cdot G_{H O_{n}}(x) & =-H O_{0} x-H O_{1} x^{2}-H O_{2} x^{3}-\cdots-H O n x^{n+1}-\cdots \\
x^{2} G_{H O_{n}}(x) & =\frac{1}{4} H O_{0} x^{2}+\frac{1}{4} H O_{1} x^{3}+\frac{1}{4} H O_{2} x^{4}+\cdots+\frac{1}{4} H O_{n} x^{n+2}+\cdots
\end{aligned}
$$

Adding each member, we have:

$$
\begin{aligned}
\left(1-x+\frac{1}{4} x^{2}\right) G_{H O_{n}}(x) & =H O_{0}+\left(H O_{1}-H O_{0}\right) x+\left(H O_{2}-H O_{1}+\frac{1}{4} H O_{0}\right) x^{2} \\
\left(1-x+\frac{1}{4} x^{2}\right) G_{H O_{n}}(x) & =H O_{0}+\left(H O_{1}-H O_{0}\right) x+0 x^{2} \\
G_{H O_{n}}(x) & =\frac{H O_{0}+\left(H O_{1}-H O_{0}\right) x}{\left(1-x+\frac{1}{4} x^{2}\right)} .
\end{aligned}
$$

Similar to the recurrence relation for the Oresme's hybrid numbers, one can present the recurrence to the negative side of these numbers.

Definition 3.8. The recurrence relation of negative integers of Oresme hybrids is defined by:

$$
H O_{n}=4\left(H O_{n+1}-H O_{n+2}\right), n<0 .
$$

From the recurrence relation of negative integers of Oresme hybrids, it is possible to obtain the first terms being: $H O_{-1}=-2+\frac{1}{2} \varepsilon+\frac{2}{4} h$ and $H O_{-2}=-8-2 i+\frac{1}{2} h$. 


\section{Conclusion}

In this work it was possible to present a study concerning the process of complexation of the Oresme sequence. This process consists of the addition of the imaginary unit $i$ and the dimensional increase of algebraic representations.

And yet it was studied, through the relation defined by Özdemir [16], the hybrid numbers of Lucas, being therefore a combination of the complex numbers, hyperbolic and the dual numbers of Lucas. It was possible to develop some properties involving this presented hybrid sequence, generating matrix, characteristic equation, norm, generating function, extension to the integer field and some identities.

The study presented highlights the importance of future studies on properties related to this sequence, as well as its application in several areas.

\section{Acknowledgements}

Part of the development of research in Brazil had the financial support of the National Council for Scientific and Technological Development (CNPq) and a Coordination for the Improvement of Higher Education Personnel (CAPES).

The research development part in Portugal is financed by National Funds through the Foundation for Science and Technology. I. P. (FCT), under the project UID/CED/00194/2020.

\section{References}

[1] Alves, F. R. V. (2019). Sequência de Oresme e algumas propriedades (matriciais) generalizadas. C.Q.D.- Revista Eletrônica Paulista de Matemática, 16, $28-52$.

[2] Bilgici, G., Tokeser, U., \& Ünal, Z. (2017). $k$-Fibonacci and $k$-Lucas Generalized Quaternions. Konuralp Journal of Mathematics, 5(2), 102-113.

[3] Catarino, P. (2019). On k-Pell hybrid numbers. Journal of Discrete Mathematical Sciences and Cryptography, 22(1), 83-89.

[4] Carvalho, C. (2019). Números híbridos e sua visualização no geogebra. Dissertação (Mestrado Profissional)- Universidade Estadual do Ceará, Centro de Ciência e Tecnologia, Mestrado Profissional em Matemática em Rede Nacional, Fortaleza.

[5] Cerda-Morales, G. (2018). Investigation of generalized hybrid Fibonacci numbers and their properties. arXiv preprint, arXiv:1806.02231.

[6] Cook, C. (2004). Some sums related to sums of Oresme numbers. In: Howard F. T. (eds) Applications of Fibonacci Numbers, Springer, Dordrecht, 87-99.

[7] Cerda-Morales, G. (2019). Oresme Polynomials and Their Derivatives. arXiv preprint arXiv:1904.01165. 
[8] Dasdemir, A., \& Bilgici, G. (2019). Gaussian Mersenne numbers and generalized Mersenne quaternions. Notes on Number Theory and Discrete Mathematics, 25(3), 87-96.

[9] Dasdemir, A. (2016). Generalizations of Modified Pell and Pell Lucas Sequences and Their Generating Matrices and Some Sums. Erzincan University Journal of Science and Technology, 9(3), 178-184.

[10] Ercolano, J. (1979). Matrix generators of Pell sequences. The Fibonacci Quarterly, 17(1), 71-77.

[11] Horadam, A. F. (1974). Oresme numbers. The Fibonacci Quarterly, 12(3), 267-271.

[12] Koshy, T. (2001). Fibonacci and Lucas Numbers with Applications. [S.l.]: New York: Wileyand Sons publications, 1.

[13] Mangueira, M. C. dos S., et al. (2020). The Hybrid Numbers of Padovan and Some Identities. Annales Mathematicae Silesianae, 34(2), 256-267.

[14] Mangueira, M. C. dos S., \& Alves, F. R. V. (2020). Números híbridos de Fibonacci e Pell. Revista Thema, 17(3), 831-842.

[15] Mangueira, M. C. dos S., Alves, F. R. V., \& Catarino, P. M. M. (2020). Números híbridos de Mersenne. C.Q.D. - Revista Eletrônica Paulista de Matemática, 18, 1-11.

[16] Özdemir, M. (2018). Introduction to hybrid numbers. Advances in Applied Clifford Algebras, 28, Art. No. 11.

[17] Sentürk, T. D., Bilgici, G., Dasdemir, A., \& Ünal, Z. (2020). A study on Horadam hybrid numbers. Turkish Journal of Mathematics, 44, 1212-1221.

[18] Sentürk, T. D., Dasdemir, A., Bilgici, G., \& Ünal, Z. (2019). On unrestricted Horadam generalized quaternions. Utilitas Mathematica, 110, 89-98.

[19] Szynal-Liana, A. (2018). The Horadam hybrid numbers. Discussiones Mathematicae General Algebra and Applications, Sciendo, 38(1), 91-98.

[20] Szynal-Liana, A., \& Wloch, I. (2019). On Jacobsthal and Jacobsthal-Lucas hybrid numbers. Annales Mathematicae Silesianae, Sciendo, 33(1), 276-283.

[21] Vieira, R., Alves, F. R., \& Catarino, P. (2020). (s1, s2)-Padovan matrix sequence and the case of generalization. Analele ştiinţifice ale Universităţii "Al. I. Cuza” din Iaşi Mat. (N.S.) (to appear). 